
allemande

46-2 | 2014

Intellectuels et politique en Allemagne

\title{
Arnold Gehlen et les mutations du conservatisme en RFA
}

Olivier Agard

\section{(2) OpenEdition}

1 Journals

Édition électronique

URL : https://journals.openedition.org/allemagne/1119

DOI : 10.4000/allemagne. 1119

ISSN : 2605-7913

Éditeur

Société d'études allemandes

Édition imprimée

Date de publication : 30 décembre 2014

Pagination : $317-330$

ISSN : 0035-0974

\section{Référence électronique}

Olivier Agard, «Arnold Gehlen et les mutations du conservatisme en RFA », Revue d'Allemagne et des

pays de langue allemande [En ligne], 46-2 | 2014, mis en ligne le 29 juillet 2019, consulté le 18 mai 2021.

URL : http://journals.openedition.org/allemagne/1119; DOI : https://doi.org/10.4000/allemagne.1119 


\section{Arnold Gehlen et les mutations du conservatisme en RFA}

\section{- Olivier Agard*}

On peut aujourd'hui accéder sur internet à l'enregistrement des débats télévisés des années 1960 entre Theodor Wiesengrund Adorno et Arnold Gehlen ${ }^{(1)}$, et le partage des rôles semble parfaitement clair: Adorno est celui qui défend les valeurs d'autonomie individuelle de l'Aufklärung face au "monde administré ", tandis que Gehlen considère que l'essentiel est de défendre les institutions contre les aspirations des individus au développement de cette autonomie, qui constitue une menace pour les institutions, et qui est pour les individus eux-mêmes une source d'insécurité et d'angoisse. Arnold Gehlen, dans ces débats (qui ont également eu lieu à la radio), assume la position du conservateur résolu. Toute une tradition de reconstruction de l'histoire intellectuelle de la RFA considère que les intellectuels de l'École de Francfort ont apporté une contribution fondamentale à la fondation intellectuelle de la République fédérale, c'est-à-dire à l'établissement d'un esprit démocratique en $\mathrm{RFA}^{(2)}$, tandis que des personnages comme Gehlen apparaissent comme les continuateurs de traditions antilibérales, antimodernes inscrites dans une longue tradition allemande. Pour Jürgen Habermas, l'institutionnalisme de Gehlen est viscéralement hostile à l'Aufklärung et perpétue, à l'image de ce que Habermas appelle "néo-conservatisme ", un Sonderweg anti-occidental de l'Allemagne, qui a conduit au national-socialisme ${ }^{(3)}$. C'était à certains égards l'image de Gehlen qu'avaient Max Horkheimer et Adorno. Un rapport d'expertise, retrouvé dans les archives de Horkheimer et intitulé " Entwurf Max »,

* Maître de conférences HDR, Université Paris IV-Sorbonne.

1 Voir en particulier le dialogue télévisé de 1965 entre Adorno et Gehlen autour du thème " Liberté et institution $»$ : http://www.youtube.com/watch?v=0o3eITHmIek\&list=PL5ocqrhdV12IopgBDWxhPK 6x7iUfhhlQp (consulté le 30 juillet 2014).

2 Clemens Albrecht, Günter C. Behrmann, Michael Bock (et al.), Die intellektuelle Gründung der Bundesrepublik: eine Wirkungsgeschichte der Frankfurter Schule, Francfort-sur-le-Main/New York, Campus, 1999.

3 Jürgen Habermas, "Die Kulturkritik der Neokonservativen in den USA und in der Bundesrepublik », in: Kleine politische Schriften, Francfort-sur-le-Main, Suhrkamp, 1981, p. 30-56. 
présente Gehlen comme un fasciste, même s'il est précisé que son fascisme se distingue du racisme völkisch ${ }^{(4)}$. Gehlen est même mis en rapport avec Ernst Krieck, le théoricien de l'éducation nationale-socialiste, pour sa valorisation du sacrifice ${ }^{(5)}$. Dans un second rapport d'expertise, Adorno et Horkheimer développent un point de vue plus nuancé et argumenté, mais qui est lui aussi sans équivoque: Gehlen produit des "idéologies de légitimation d'un État autoritaire " ${ }^{(6)}$. De fait, à première vue, l'intervention de Gehlen la plus marquante dans le débat intellectuel, son ouvrage Moral und Hypermoral ${ }^{(7)}$, paru en 1969, semble aller dans le sens du portrait dressé par ses adversaires de gauche: Gehlen y fustige les intellectuels, critique l'État providence dépolitisé qui transforme le Léviathan en "vache à lait », dénonce la morale humanitaire qui a envahi l'espace public et suggère que ce moralisme est utilisé comme une arme contre l'Allemagne vaincue (dont la situation se voit comparée à celle d'Athènes après la guerre du Péloponnèse). Il reçoit à l'époque le soutien d'Armin Mohler, figure de la nouvelle droite, et de la revue Criticon.

Toutefois, même si le positionnement conservateur de Gehlen ne fait pas de doute, les choses sont plus compliquées qu'une présentation binaire de l'histoire intellectuelle de la RFA pourrait le laisser penser. Ainsi, Adorno et Horkheimer sont loin de s'identifier sans partage aux institutions parlementaires de la RFA, ou à l'économie sociale de marché. La tradition de critique de la civilisation moderne (Kulturkritik) a laissé des traces profondes chez Adorno, et c'est un terrain où - au moins sur le plan de la description des pathologies de la société de masse - il n'est pas si loin de Gehlen, ce qui explique la relation plutôt amicale qu'il a pu entretenir avec lui ${ }^{(8)}$. Symétriquement, un livre récent développe une thèse stimulante selon laquelle il a existé dès la fin des années 1960 un " conservatisme libéral », qui s'est élaboré au sein du cercle du philosophe Joachim Ritter à Münster et qui rassemble des philosophes comme Hermann Lübbe, Robert Spaemann, Odo Marquardt. Ce conservatisme libéral aurait eu une fonction stabilisatrice de légitimation des institutions de la RFA ${ }^{(9)}$. Si Gehlen n'appartient pas à ce courant, sa théorie des institutions est un des éléments de la philosophie politique de ces auteurs, qui finit par déboucher sur une défense de la démocratie parlementaire dans sa forme existante, mais à partir de présupposés qui ne sont pas ceux du libéralisme anglo-saxon ou du républicanisme français.

Parler du conservatisme de Gehlen est pertinent, mais n'est pas suffisant, compte tenu du flou qui entoure cette notion. Trop souvent, dans le débat idéologique et médiatique, on identifie pensée conservatrice et pensée réactionnaire. Si l'on définit

4 Voir: Theodor W. Adorno - Max Horkheimer, Briefwechsel, vol. IV : 1950-1969, Francfort-sur-leMain, Suhrkamp, 2006, p. 927.

5 Ibid., p. 928. Adorno et Horkheimer incriminent une phrase qui est en réalité une citation de Winston Churchill (ce qu'ils ne signalent pas).

6 Ibid., p. 931.

7 Arnold Gehlen, Moral und Hypermoral, eine pluralistische Ethik (1969), Francfort-sur-le-Main, Athenäum, 1970.

8 Christian Thies, Die Krise des Individuums: zur Kritik der Moderne bei Adorno und Gehlen, Reinbek bei Hamburg, Rowohlt, 1997.

9 Jens Hacke, Philosophie der Bürgerlichkeit: die liberalkonservative Begründung der Bundesrepublik, Göttingen, Vandenhoeck \& Ruprecht, 2008. 
simplement le conservatisme comme un rejet de la modernité politique et sociale, on est obligé de considérer comme Panajotis Kondylis qu'il n'y a plus de véritables conservateurs depuis la disparition des derniers légitimistes ${ }^{(10)}$. Si l'on veut sortir de cette aporie, il faut plutôt définir le conservatisme comme une stratégie d'adaptation. Comme le rappelle Heinrich Lübbe, le conservatisme ne peut de ce point de vue naître qu'au sein de la modernité, il suppose le sentiment d'une histoire en marche ${ }^{(11)}$. Le conservatisme est donc fondamentalement un concept relationnel, et on ne peut pas en définir une doctrine cohérente. Le but de la présente contribution est de montrer que - dans le contexte de la RFA - le conservatisme de Gehlen n'est pas celui d'Armin Mohler, qui correspond davantage à la vision qu'a Habermas du conservatisme: la persistance d'une tradition d'opposition aux valeurs de l'Aufklärung " occidentale ", et la perpétuation d'un héritage venu en droite ligne de la "révolution conservatrice». Gehlen a gardé ses distances vis-à-vis de ces cercles, et c'est la raison pour laquelle il a été considéré comme fréquentable par le système médiatique libéral: il a publié dans Merkur ou dans Westermann's Monatshefte, et fut régulièrement invité à la radio et à la télévision (notamment pour débattre avec Adorno). Il ne s'agit toutefois pas non plus de soutenir qu'il appartenait au conservatisme libéral évoqué par Jens Hacke: en effet, Gehlen reste un critique résolu de l'idéal d'autonomie individuelle issue de l'Aufklärung.

Quoi qu'il en soit, pour comprendre le conservatisme de Gehlen, il est essentiel de prendre en compte sa construction philosophique. C'est ce qui permet d'en saisir la nature assez atypique: en effet, il s'agit d'un conservatisme paradoxal, parce qu'il ne repose pas sur une vision statique de l'homme et de la société. Il ne résulte pas non plus, comme souvent en Allemagne, d'une philosophie de l'historisme qui valoriserait la tradition en tant que telle (on retrouve en revanche cette philosophie " historiste " dans le " conservatisme libéral »). Au cœur du conservatisme de Gehlen, se trouve l'idée que l'homme est foncièrement mobile et instable. D'un certain point de vue, il existe une tension entre l'anthropologie de Gehlen et sa politique, ou, du moins, certains potentiels de son anthropologie sont-ils court-circuités par des choix politiques et idéologiques qui ne découlent pas nécessairement des prémisses de son anthropologie.

\section{L'instabilité structurelle de l'homme et le paradigme de l'action}

Sans entrer ici dans les détails de l'anthropologie de Gehlen, on peut dire qu'il s'agit pour lui, comme pour l'anthropologie philosophique allemande en général (entendue ici comme un courant de pensée spécifique $\left.{ }^{(12)}\right)$, de partir de la situation de l'homme dans la vie. Gehlen constate en effet un hiatus entre l'homme et son milieu: l'homme est une «créature lacunaire» («Mängelwesen ») pauvre en instincts, qui n’est pas suffisamment dotée biologiquement pour s'adapter spontanément à un environnement

10 Panajotis Kondylis, Konservatismus: geschichtlicher Gehalt und Untergang, Stuttgart, Klett-Kotta, 1986.

11 Cf. le commentaire de J. Hacke, Philosophie der Bürgerlichkeit (note 9), p. 132.

12 Cf. Joachim Fischer, Philosophische Anthropologie: Eine Denkrichtung des 20. Jahrhunderts, Fribourgen-Brisgau, Alber, 2008. 
donné. Cette situation présente des avantages et des inconvénients: elle fait de l'homme un être qui doit se construire lui-même, doit agir de façon créatrice sur lui-même et son milieu. Par ailleurs, elle le confronte à un « excédent pulsionnel » ("Triebüberschuss »): son énergie n'est pas spontanément régulée, mais doit être canalisée. La culture a donc une double fonction: celle de permettre à l'homme de vivre dans son environnement par le biais d'une action créatrice de transformation active de lui-même et de son environnement (action qui n'est pas que simple adaptation), et en même temps celle de réguler l'excédent pulsionnel. L'homme ne peut survivre que par la création de procédures, de dispositifs, d'institutions, qui lui permettent de se soulager du poids que font peser sur lui les contraintes d'un milieu qui ne lui est pas spontanément corrélé, ainsi que les contraintes venues de sa propre nature, c'est-à-dire ses pulsions, qui ne sont pas spontanément réglées, comme chez les animaux. C’est pourquoi Gehlen définit la culture humaine comme « délestage » ( Entlastung»).

Le concept fondamental de cette anthropologie est celui de l'action: l'homme est la seule créature capable d'agir créateur. Par cette conception, Gehlen tente de dépasser la conception idéaliste du sujet, qui oppose le corps et l'âme, l'esprit et la matière, la conscience et la réalité. L'action unifie tous ces moments. Cette conception de l'action est déjà sous-jacente dans les premiers travaux de Gehlen, alimentés par la philosophie de l'existence, la philosophie de la vie, et par un certain fichteanisme (en particulier dans Wirklicher und unwirklicher Geist en $1931^{(13)}$, et Théorie der Willensfreiheit en $1933^{(14)}$. Mais, à partir du milieu des années 1930, Gehlen réoriente sa pensée vers une théorie anthropologique de l'action. Il présente son anthropologie philosophique sous une forme développée dans Der Mensch (1940) ${ }^{(15)}$.

Si l'on s'interroge sur les prolongements culturels et politiques de cette philosophie de la volonté et de l'action, il me semble que l'on peut difficilement nier de la part de Gehlen une intention anti-libérale, au sens large. L'action résolue apparaît comme un antidote au subjectivisme et à l'intellectualisme, des pathologies implicitement associées à la démocratie libérale dans le champ discursif de l'époque. Gehlen explique ainsi dans sa théorie de la volonté que la véritable liberté n'est pas l'autonomie individuelle, mais l'adhésion au destin, à la nécessité. L'action s'oppose de ce point de vue au normativisme juridique, et à la culture de la délibération et du consensus. Le sentiment d'une crise moderne du désenchantement débouche donc chez Gehlen sur une forme de décisionnisme, et sa position relève globalement d'un réalisme héroïque, attitude qu'il avait théorisée dès 1925 dans un discours sur Hofmannstahl, dans lequel il expliquait que l'action héroïque était le seul antidote à la résignation ${ }^{(16)}$. La philosophie du national-socialisme, qu'il projette d'écrire en 1932-1935, devait prolonger la théorie de la liberté du vouloir, et valoriser la Weltanschauung nazie, comme aboutissement de la

13 Arnold GeHLen, Wirklicher und unwirklicher Geist: eine philosophische Untersuchung in der Methode absoluter Phänomenologie, Leipzig, Noske, 1931.

14 Arnold Gehlen, Theorie der Willensfreiheit, Berlin, Junker und Dünnhaupt, 1933.

15 L'œuvre connaîtra plusieurs versions. Pour une vue d'ensemble: Arnold GeHLen, Der Mensch: seine Natur und seine Stellung in der Welt (édition critique incluant le texte original de 1940) (Euvres complètes 3), Francfort-sur-le-Main, Klostermann, 1993.

16 Arnold Gehlen, « Rede über Hofmannstahl », in: A. Gehlen, Philosophische Schriften: Philosophische Schriften I (1925-1933) (Euvres complètes 1), Francfort-sur-le-Main, Klostermann, 1978, p. 1-17. 
pensée allemande. Gehlen voulait en particulier mettre en valeur la conception nationale-socialiste de l'État - un État vu comme expression du peuple et « ordre concret de l'existence » ("konkrete Daseinsordnung») - ce qui fait de ce texte une première étape vers une théorie des institutions (qui emprunte des éléments à Carl Schmitt). Dans Der Mensch, en 1940, Gehlen faisait l'éloge du national-socialisme comme exemple de "Führungssystem ", de "système directeur ", apte à fournir une vision du monde aux individus. Sans vouloir du tout minimiser l'engagement nazi de Gehlen ${ }^{(17)}$, il faut relever que son anthropologie n'a que peu de rapport avec le social-darwinisme völkisch qui est à la base de la politique impérialiste et génocidaire du national-socialisme. Gehlen parle dans Der Mensch de l'homme en général, indépendamment de toute considération sur les races (et s'il a bien eu le projet d'écrire un texte sur les races, il s'est montré incapable d'aller au bout de ce projet). Son anthropologie a d'autres sources: freudisme, bergsonisme, biologie anti-darwinienne, et surtout pragmatisme. Cela ne fait évidemment pas de Gehlen un humaniste démocrate: même si le pragmatisme est dans sa version anglo-saxonne plutôt d'inspiration démocratique, il connaît de fait en Allemagne une réception autoritaire, avec l'ouvrage d'Eduard Baumgarten ${ }^{(18)}$, réception de laquelle Gehlen et Helmut Schelsky participent ${ }^{(19)}$. Pour Baumgarten, la démocratie américaine, inspirée par le pragmatisme, n’a en effet rien à voir avec le système «ploutocratique " de Weimar ou avec l'égalitarisme français, mais a des racines germaniques ${ }^{(20)}$ : l'égalitarisme inhérent au pragmatisme a une dimension " qualitative » et "dynamique » et non pas « statique » ou " quantitative ». Le pragmatisme de Gehlen est donc bien différent de celui de William James, John Dewey ou George Herbert Mead. Comme le note à juste titre Patrick Wöhrle, les mêmes motifs pragmatiques peuvent avoir selon les contextes de réception un arrière-plan et une motivation très différents. Par exemple, quand Gehlen met en avant l'action, c'est avec une intention polémique - dirigée contre la subjectivité conçue comme intériorité et réflexion - qu'on ne trouve pas chez Dewey et Mead ${ }^{(21)}$, qui ne partagent pas la critique de la civilisation moderne à laquelle se livre Gehlen dans une bonne partie de ses écrits. Dans le pragmatisme de James, l'action est avant tout l'ouverture de nouveaux possibles dans un monde considéré comme inachevé. Pour Gehlen, l'action est certes créatrice, parce que l'homme est un animal ouvert qui s'invente lui-même et invente son monde, mais cette ouverture est pensée sous l'angle d'une fragilité angoissante qui suscite un besoin de clôture, de canalisation, d'habitude: et si l'action est créatrice en son principe, sa

17 Voir à ce sujet, par exemple: Christian Thies, Gehlen zur Einführung, Hambourg, Junius, 2000, p. 15-17.

18 Eduard Baumgarten, Die Geistigen Grundlagen des amerikanischen Gemeinwesens, Francfort-sur-leMain, Klostermann, 1936-1938, vol. I: Benjamin Franklin, der Lehrmeister der amerikanischen Revolution (1936); vol. II : Der Pragmatismus: R. W. Emerson, W. James, J. Dewey (1938). Sur la réception «fasciste» du pragmatisme: Walter GAGEL, Geschichte der politischen Bildung in der Bundesrepublik Deutschland 1945-1989/90, Wiesbaden, Verlag für Sozialwissenschaften, 2005, p. 65-68, ainsi que: Peter Vogt, Pragmatismus und Faschismus; Kreativität und Kontingenz in der Moderne, Weilerswist, Velbrück Wiss, 2002.

19 Voir par exemple la référence au pragmatisme dans l'introduction à: Helmut SCHELSKy, Thomas Hobbes, eine politische Lehre, Berlin (Ouest), Duncker und Humblot, 1981.

20 E. Baumgarten, Der Pragmatismus (note 18), p. 324.

21 Patrick Wöhrle, Metamorphosen des Mängelwesens: zu Werk und Wirkung Arnold Gehlens, Francfort-sur-le-Main, Campus Verlag, 2010, p. 172. 
finalité est la stabilisation. C'est tout le paradoxe de la conception de Gehlen, qui ne partage pas l'optimisme mélioriste d'un William James, parce que l'instabilité est chez Gehlen une menace, un danger, même si elle est la condition de la créativité et de la liberté de l'homme. Ce pragmatisme singulier donne au plaidoyer de Gehlen pour le national-socialisme une coloration particulière. Si le national-socialisme se voit justifié en tant que "système directeur ", c'est en raison de son rôle stabilisateur plutôt que pour son contenu historique réel, et la vérité de sa vision du monde: ce système directeur offre une solution au problème structurel de l'instabilité humaine. C'est pourquoi l'ouvrage a suscité certaines réserves à l'époque de sa parution: il ne développait en effet pas une philosophie de l'histoire optimiste et conquérante. Il ne s'agit évidemment pas de nier l'engagement national-socialiste de Gehlen ni de dissimuler le fait que sa pensée n'offre pas d'argument à opposer au national-socialisme: cette absence de point de vue normatif est comme on le verra un problème majeur de la théorie des institutions de Gehlen, même s'il donne lui-même quelques pistes ${ }^{(22)}$.

\section{Fondement et crise des institutions}

Si Gehlen insiste dans la première version de Der Mensch plutôt sur la nécessité d'une vision du monde (celle-ci permettant aux hommes de s'orienter et de se mobiliser dans leur monde et de lui conférer un sens), il est conduit dans son œuvre ultérieure à s'interroger sur les institutions, et il faut sans doute y voir une conséquence de l'expérience du nazisme. La thématique de la stabilité institutionnelle (incarnée par l'Église, l'armée, la famille, etc.) est mise au premier plan, au détriment du décisionnisme et de l'apologie d'une vision du monde mobilisatrice. Cette théorie des institutions est la base du conservatisme de Gehlen des années 1950 et 1960. Toutefois, cette théorie des institutions est traversée par le même paradoxe que l'anthropologie, la même dialectique de la stabilité et de l'instabilité, ce qui n'est pas étonnant, car dans son ouvrage Urmensch und Spätkultur (paru en 1956 et conçu comme le volume II de Der Mensch), Gehlen relie la théorie des institutions à son anthropologie et à sa théorie de l'action, en nuançant et différenciant cette théorie (qui distingue désormais différents types d'action) ${ }^{(23)}$. C'est en effet dans le hiatus entre l'homme et son environnement que vient s'insérer l'institution, qui installe l'homme dans le monde stabilisé de la culture, et le « déleste » de son instabilité première. L'institution a donc pour Gehlen un double visage: elle est à la fois ouverture créatrice et stabilisation contraignante. En effet, il importe à Gehlen de montrer dans cet ouvrage que l'institution est l'expression de l'agir créateur de l'homme, c'est-à-dire de sa capacité à prendre ses distances par rapport aux contraintes naturelles (internes et externes) qui pèsent sur lui. En ce sens, l'institution est aussi l'expression et la condition de la liberté, celle-ci résultant d'une tension stabilisée, qui dirige l'énergie des pulsions vers des objectifs sociaux (le totémisme apparaissant comme un moment clé dans la réalisation de cette liberté). Un enjeu de l'ouvrage est à cet égard de réfuter une interprétation simplement fonctionnaliste des institutions: les institutions ne sont pas un mécanisme purement réactif d'adaptation

22 Comme le rappelle C. Thies, Gehlen (note 17), p. 128-129.

23 Arnold GeHLen, Urmensch und Spätkultur: philosophische Ergebnisse und Aussagen (1956), Francfortsur-le-Main, Klostermann, 2004. 
et de régulation. Gehlen met en valeur la capacité positive de création, qui fait que la culture dépasse d'emblée le simple système des besoins et la nécessité de l'autoconservation, même si elle commence par la création d'instruments (qui mettent toutefois en œuvre dès le départ une capacité d'imagination). Les institutions sont le produit d'une conscience "idéative », et sur ce point, Gehlen s'inspire du juriste français Maurice Hauriou et de sa notion d'" idée directrice ». Pour Hauriou, les institutions du droit ou de l'État ne sont pas le produit d'un processus social désincarné, ou d'un système de normes, qui se déploierait au-dessus de la tête des individus, et qui ne «tirerait sa force » que du «substrat social » dont elles émanent - comme le font chez Durkheim les dogmes, les rites ou la religion, ce qui conduit à l'idée que ceux-ci n'ont plus qu'une "valeur d'emprunt ( (selon un commentaire de Célestin Bouglé ${ }^{(24)}$ ). Pour Hauriou, les institutions naissent de la conjonction d'une "idée directrice " et d'un intérêt vital, elles sont le produit d'une communion, d'un acte créateur. Carl Schmitt utilisait cette théorie dans le cadre d'une critique de la conception normativiste et abstraite du droit. Gehlen l'intègre pour sa part dans une théorie anthropologique des institutions, qui partage la même orientation anti-normativiste et anti-libérale, mais considère, à la différence de Schmitt, que la perte des idées directrices est un phénomène irréversible. Par ailleurs, chez lui, cette notion d'idée directrice, qui a sa source dans une sociologie vitaliste inspirée d'Henri Bergson, ne saisit qu'un aspect de la réalité de l'institution. En effet, c'est malgré tout in fine la fonction de stabilisation sur laquelle Gehlen insiste toujours et encore: la créativité, l'imagination passent au second plan au profit de la création d'habitudes; Gehlen finit donc par faire - d'une certaine façon - l'apologie de ce que Bergson appelle dans Les deux sources de la morale et de la religion (1932) la société « close » (même si la stabilisation institutionnelle est pour Gehlen autre chose qu'un carcan purement mécanique et normatif). Or Hauriou, s'inspirant de Bergson, voulait réintroduire dans la théorie du droit une dimension de vitalité créatrice. Alors que Bergson, qui, il est vrai, ne se place pas réellement sur le plan d'une théorie des institutions, entrevoit la possibilité d'une régénération de la société et de la morale " closes " par la morale « ouverte ", plus proche de l'élan créateur de la vie, et incarnée par les grands mystiques, Gehlen, qui connaît cette théorie bergsonienne évoquée à la fin de Moral und Hypermoral ${ }^{(25)}$, élabore un scénario plus tragique et pessimiste. Les institutions sont pour lui condamnées à s'éroder et à perdre leur pouvoir stabilisateur, parce qu'en permettant aux individus de se décharger des contraintes matérielles, elles ouvrent un espace au développement de la subjectivité, un développement qui vient réintroduire de l'instabilité. C'est ici qu'il faut situer - sur un plan fondamental - le moment conservateur de la pensée de Gehlen. Il réside dans cette philosophie inquiète qui voit le développement de l'autonomie individuelle comme une menace pour les institutions. Significativement dans ce scénario de dépérissement des institutions, l'Aufklärung constitue une étape majeure, même si pour Gehlen, celui-ci commence avec le monothéisme. Cette polémique contre l'Aufklärung est développée dans Moral und Hypermoral où il est dit: "En bref, l'Aufklärung est l'émancipation de l'Esprit hors des institutions » («Die Aufklärung ist, kurz gesagt, die Emanzipation

24 Célestin Bouglé, Bilan de la sociologie française contemporaine, Paris, Alcan, 1935, p. 162.

25 A. Gehlen, Moral und Hypermoral (note 7), p. 168-170. 
des Geistes von den Institutionen ") ${ }^{(26)}$. On peut toutefois noter que Gehlen aurait pu trouver dans sa propre anthropologie des ressources pour un scénario alternatif. On pourrait imaginer que les institutions soient revivifiées par un acte collectif créateur, ou qu'il y ait une action en retour de l'individu sur l'institution, une participation de l'individu à la vie des institutions, une sorte de dialectique entre création et stabilisation. Lorsqu'il évoque l'homme acteur de lui-même et de son milieu, Gehlen consacre tout un passage à l'imagination ("Phantasie»): pourquoi ne pas considérer que cette imagination pourrait être mise en œuvre pour faire évoluer les institutions? Gehlen refuse d'entrevoir cette possibilité, et considère qu'avec le désenchantement du monde, l'homme est de plus en plus un consommateur, un individu guidé par ses désirs privés, et qu'il est voué à perdre ses capacités créatrices: l'individualisme moderne ne peut conduire qu'à la fin de la personnalité créatrice dont l'institution a besoin pour sa naissance et sa perpétuation.

Pour les auteurs pragmatistes anglo-saxons, il n'y a pas d'antagonisme entre créativité et développement des droits de l'individu. C'est pourquoi Dewey applique sa théorie de l'agir créatif au niveau des institutions (la société et les institutions doivent pour lui obéir également à une logique créative et expérimentale) ${ }^{(27)}$. Selon Hans Joas, il y a là un différend entre la tradition américaine et une certaine tradition allemande: dans la tradition américaine, la pensée créatrice n'est pas perçue comme contraire à la démocratie libérale, alors qu'en Allemagne, la créativité est plutôt associée à une sorte d'opposition esthétique à la modernité(28). Chez Gehlen, comme chez Carl Schmitt, l'individualisme est vu comme une force dissolvante et dangereuse. Pour ce dernier, la valorisation de la sphère individuelle est le point faible de la théorie de Hobbes, la brèche dans laquelle s'est infiltré Spinoza et qui conduit au libéralisme moderne. À la différence des auteurs proches de la révolution conservatrice, Gehlen ne pense toutefois pas que la solution au problème qui renvoie à un phénomène irréversible soit une régénération substitutive du Volk. C’est en ce sens que cette critique du dépérissement des institutions relève davantage d'une sociologie pessimiste de la culture, d'une Kulturkritik, que d'une philosophie politique qui offrirait une alternative. C'est pourquoi cette théorie des institutions, malgré son opposition aux valeurs qui sont supposées inspirer l'ordre politique et économique de la RFA n'a pas la même toxicité politique que le discours de la révolution conservatrice à l'époque de la République de Weimar. Par un nouveau paradoxe, cette théorie finit par légitimer une position plutôt défensive de légitimation de l'ordre existant. Comme on le disait au départ, le conservatisme est un concept réactif. Il s'agit surtout pour Gehlen de s'opposer aux tentatives de valoriser le développement de l'individu, de remettre en cause les structures institutionnelles, de développer les contre-pouvoirs. Il s'agit aussi de contrer le pôle intellectuel que constitue l'École de Francfort, qui alimente une critique sociale émancipatrice.

26 Ibid., p. 102.

27 Cf. P. Wöhrle, Metamorphosen des Mängelwesens (note 21), p. 164. 


\section{Le réalisme technocratique et ses prolongements}

La légitimation défensive de l'existant prend chez Gehlen la forme de ce qu'on pourrait appeler le réalisme technocratique. Sur un plan fondamental, sa théorie des institutions était de nature à alimenter dans le contexte de la RFA une critique radicale vis-à-vis de la démocratie libérale, de la technocratie, de la société de masses, de l'économie de marché. Tous ces phénomènes vont pour Gehlen dans le sens d'un affaiblissement des institutions. Le règne de la technostructure est à ses yeux celui de l'organisation, et l'organisation n'est pas une institution au sens fort du terme (elle est dépourvue d'"idée directrice »). Dans la caractérisation de cette société administrée où règne un individualisme de masse, il y a des parentés étonnantes avec le diagnostic d'Adorno, et cette parenté a été analysée par Christian Thies, qui met en avant trois grands thèmes communs qui sont: la perte de l'expérience ("Erfahrungsverlust»), la massification ("Vermassung»), et le déclin et la destruction de l'individualité ("Verfall und Zerfall der Individualität ») ${ }^{(29)}$. Mais en même temps, pour Gehlen, les organisations sont malgré tout les derniers éléments de cohésion qui restent, face au triomphe de l'individu instable régi par ses désirs consuméristes et son narcissisme. Par ailleurs, l'organisation offre une niche à la personnalité créatrice: elle peut trouver sa place au sein de cette organisation, celle de celui qui pilote le processus, le super-entrepreneur, le « super-routinier» ("Über-routinier»), le super-fonctionnaire qui cultive une distance aristocratique, et combine ascétisme et raffinement. La stratégie de Gehlen est de défendre ce qui peut être défendu dans un monde où le déclin de l'institution est programmé. Pour Gehlen, le monde moderne est en désordre, et tout ce qui menace le semblant d'autorité qui structure encore la société doit être combattu. On est en présence d'un conservatisme d'adaptation et de réaction. Il s'agit en même temps de contester - sur un plan fondamental - les prémisses de la théorie critique telle qu'elle s'implante à l'époque dans le paysage intellectuel de la RFA. À cette fin, Gehlen insiste sur un antagonisme réel entre théorie critique et anthropologie philosophique: alors que la théorie critique cherche les voies d'une sortie de l'aliénation, d'une émancipation (même si l'aliénation est un moment nécessaire), Gehlen met en valeur le fait que la condition humaine est structurellement marquée par une aliénation indépassable, qui est le principe anthropologique de sa liberté, comme il l'explique dans un texte au titre significatif: "Naissance de la liberté à partir de l'esprit de l'aliénation » (Über die Geburt der Freiheit aus dem Geist der Entfremdung " ${ }^{(30)}$ ). En ce sens, ce que la théorie critique voit comme une réification, le développement d'un monde dont l'individu est dépossédé, et qu'il doit se réapproprier, est pour Gehlen un phénomène positif: Gehlen décrit ce phénomène en termes d'autonomisation («Verselbständigung»). Une différence fondamentale entre Adorno et Gehlen est donc que ce qui apparaît au premier comme Entfremdung ou aliénation, c'est-à-dire comme un état qui ne permet pas aux sujets de réaliser leurs potentiels de créativité et de bonheur, est pour l'autre Entlastung (" délestage »). La critique émancipatrice de l’aliénation développée par

29 C. Thies, Die Krise des Individuums (note 8).

30 Arnold Gehlen, «Über die Geburt der Freiheit aus dem Geist der Entfremdung », in: Philosophische Anthropologie und Handlungslehre (Euvres complètes 4), Francfort-sur-le-Main, Klostermann, 1983, p. 366-379. 
l'École de Francfort paraît à Gehlen reposer sur une anthropologie utopique, la vision d'une humanité qui pourrait réaliser son essence (qui serait donc réprimée dans les structures sociales existantes). C'est ce moment utopique que Gehlen reproche à Adorno dans un de leurs entretiens radiophoniques, lorsqu'il lui demande: "Mais comment connaissez-vous le potentiel non contrôlé qui existe dans les hommes?» («Woher wissen Sie denn, was als Potential in den Menschen ungelenkt steckt? »). Adorno répond qu'il ne connaît ce potentiel que de façon négative, par la souffrance que suscite sa non-réalisation ${ }^{(31)}$. De même, selon Gehlen, on ne peut exiger de tout le monde qu'il accède au degré de conscience et de réflexivité du philosophe, c'est-à-dire qu'il porte la " charge » ("Belastung») de la réflexion, ce à quoi Adorno répond qu'il faut avoir cette exigence. Gehlen remarque alors: « Nous en sommes exactement arrivés au point où vous dites "oui" et où je dis "non” » (32) ("Da sind wir nun genau an dem Punkt, wo Sie “ja” sagen und ich "nein” »). En même temps, Gehlen construit sur la base de cette divergence un antagonisme radical entre autonomie individuelle et liberté : la vraie liberté, celle de l'agir créateur n'est possible que lorsque le sujet s'identifie à l'institution. Gehlen radicalise ainsi une tradition d'hégélianisme de droite (qui s'oppose à l'hégélianisme de gauche de l'École de Francfort), en posant que le sujet ne peut exister véritablement que dans et à travers l'institution. Pour justifier l'impossibilité d'une refondation des institutions, il est contraint de recourir à la critique de la civilisation, et d'expliquer que nous sommes entrés dans la post-histoire, c'est-à-dire dans une ère de "cristallisation culturelle », de fin de la créativité humaine: il n'y aura plus de nouvelle grande vision du monde. Le conservatisme technocratique de Gehlen est donc teinté de résignation et de mélancolie. En dépit de son réalisme affiché, il est étroitement lié à une Kulturkritik dont il prétend s'affranchir (par exemple lorsqu'au début de Die Seele im technischen Zeitalter (1957), Gehlen remet en cause la tradition de la critique de la technique, au nom de l'idée que la technique est aussi vieille que l'homme lui-même et le définit) ${ }^{(33)}$. La position de Gehlen pouvait dans le contexte des années 1960 susciter le reproche d'entretenir une « mentalité autoritaire » au cœur de la démocratie, et alimenter le doute des intellectuels progressistes et des étudiants baby-boomers sur les arrière-pensées des élites administratives et économiques de la RFA: Gehlen, d'abord enseignant à l'école supérieure d'administration de Spire, puis à l'université technique d'Aix-la-Chapelle, était en effet présent dans le conseil scientifique de plusieurs institutions liées au patronat (comme la fondation Walther Raymond Stiftung). De façon générale, il est frappant de voir que le conservatisme technocratique était souvent propagé par des penseurs compromis dans le national-socialisme. Il

31 «Il est vrai que je ne sais pas de façon positive ce qu'est ce potentiel, mais à partir de toutes sortes de perspectives partielles [...], je sais que les processus d'adaptation auxquels les hommes sont soumis vont dans le sens d'une mutilation des hommes, dans des proportions indescriptibles... ", Friedemann Grenz, Adornos Philosophie in Grundbegriffen: Auflösung einiger Deutungsprobleme. Mit einem Anhang "Ist die Soziologie eine Wissenschaft vom Menschen? Ein Streitgespräch ", Francfort-sur-le-Main, Suhrkamp, 1974, p. 246. ( $~ N a$, das weiß ich zwar nicht positiv, was dieses Potential ist, aber ich weiß aus allen möglichen [...] Teileinsichten, daß die Anpassungsprozesse, denen die Menschen heute gerade unterworfen sind, in einem unbeschreiblichen Umfang [...] auf Verkrüppelung der Menschen hinauslaufen. ») Ibid., p. 250.

33 Arnold GeHLen, Die Seele im technischen Zeitalter und andere sozialpsychologische, soziologische und kulturanalytische Schriften (Euvres complètes 6), Francfort-sur-le-Main, Klostermann, 2004. 
est toutefois à noter que dans son entretien avec Adorno sur la liberté et l'institution, Gehlen récuse l'idée que l'aspiration à des institutions fortes et indiscutées serait l'expression d'une personnalité autoritaire, et appelle plutôt au besoin de protection et à la vulnérabilité de l'individu: c'est toujours l'idée que l'instabilité de l'homme est une position lourde de menaces et pleine de dangers. Il faut reconnaître qu'il s'agit là d'une autre anthropologie que celle de Carl Schmitt, qui part plutôt de l'hostilité et de la conflictualité comme fait premier des relations entre les hommes. Mais lorsqu'il met en avant les dangers liés à la position de l'homme dans la vie, et en déduit le besoin d'institutions stables, Gehlen court-circuite un autre développement possible de cette anthropologie: il pourrait en effet en tirer la conséquence d'un besoin de solidarité et de protection, et c'est un peu dans ce sens que Hans Blumenberg s'efforcera d'orienter l'anthropologie philosophique ${ }^{(34)}$. Gehlen fait d'ailleurs dans Moral und Hypermoral une place à l'éthique de la solidarité, ancrée selon lui dans la famille, mais il déplore la place excessive qu'elle aurait prise à l'ère de l'humanitarisme et de l'État providence. L'anthropologie philosophique ne débouche donc pas nécessairement sur une approche conservatrice de la société, même si par rapport à la théorie critique, elle renonce à l'utopie d'une humanité et d'un individu non aliénés. L'œuvre de Helmuth Plessner (pratiquement contemporaine de celle de Gehlen) illustre cette possibilité d'une version plus démocratique de l'anthropologie philosophique.

Même si Gehlen s'inquiétait dans la RFA des années 1960 de la montée de contrepouvoirs et des aspirations de la société à peser sur la politique, on pourrait défendre l'idée que ce réalisme technocratique a malgré tout, à son corps défendant, accompagné la démocratisation de la RFA, en permettant la transition d'un conservatisme völkisch et nationaliste, politiquement agressif, vers un conservatisme dépolitisé et résigné, compatible avec le parlementarisme et la construction européenne. L'intégration de Gehlen par le système médiatique dans les années 1960 est un indice de cette fonction de transition intégratrice: Gehlen s'est retrouvé dans la position d'un critique des médias de masse qui entre dans le jeu qu'il prétend dénoncer. La Kulturkritik des années 1950 a eu cette même fonction d'intégration du champ intellectuel et d'accompagnement résigné de l'évolution vers le parlementarisme et le libéralisme. Comme l'a montré Georg Bollenbeck, si la Kulturkritik a de fait connu un grand écho dans les années $1950^{(35)}$ - comme l'atteste le succès de Die Seele im technischen Zeitalter -, c'est parce qu'elle a canalisé, en la dépolitisant, une opposition radicale, " anti-moderne » au capitalisme libéral (qu'elle soit de droite ou de gauche). La Kulturkritik était une figure de repli commode, d'autant plus qu'elle avait l'avantage d'ouvrir un espace commun à la droite et à la gauche (comme le montrent les convergences entre Gehlen et Adorno) ${ }^{(36)}$.

34 Jean-Claude Monod, "L'interdit anthropologique” chez Husserl et Heidegger et sa transgression par Husserl et Heidegger ", in: Olivier Agard, Céline Trautmann-Waller (dir.), L'anthropologie allemande entre philosophie et sciences, des lumières aux années 1930, Revue Germanique Internationale, 10, Paris, CNRS éditions, 2009, p. 221-236.

35 Georg Bollenbeck, Gerhard Kaiser (dir.), Die janusköpfigen 50er Jahre: kulturelle Moderne und bildungsbürgerliche Semantik III, Wiesbaden, Westdeutscher Verlag, 2000.

36 Sur cette dimension intégratrice de la Kulturkritik: Thomas Keller, "Kulturkritik nach 1945 », in: Gilbert Merlio, Gérard Raulet (dir.), Linke und rechte Kulturkritik: Interdiskursivität als Krisenbewusstsein, Francfort-sur-le-Main, Lang, 2005, p. 307. 


\section{Après Gehlen}

Mais si Gehlen est sans doute à son corps défendant un maillon dans l'évolution d'un certain conservatisme vers un soutien relatif aux institutions de la RFA, son discours restait trop ambivalent: sa justification défensive de l'ordre en tant que tel n'offrait pas de critère permettant d'apporter une légitimité positive à la démocratie libérale. Dans une décennie - les années 1960 - marquée par des mouvements d'émancipation, en Allemagne et dans le monde, cette stratégie d'adaptation montrait ses limites, ce qu'a bien compris une partie des conservateurs, en particulier après le tournant de 1968, et les « années de plomb » qui conduisirent à une restructuration du courant conservateur, et, si l'on suit Jens Hacke, à l'émergence d'un conservatisme libéral, dans lequel vinrent s'insérer des intellectuels plutôt venus de la gauche modérée. La reprise des théories de Gehlen par un discours plus affirmatif quant à la réalité de la RFA ne pouvait se faire qu'au prix d'une correction de cette théorie. Un premier type de correction est celui qu'apporte Helmut Schelsky, qui, bien que venant d'un horizon intellectuel proche de celui de Gehlen, considère que sa critique de la subjectivité va trop loin. Schelsky a très bien identifié le noyau hyper-conservateur de la pensée de Gehlen, c'est-à-dire cette opposition rigide entre individu autonome et institution (qui renvoie à une antithèse tout aussi rigide entre action et réflexion) et il tente de montrer que l'autonomie individuelle n'est pas nécessairement l'ennemie du fonctionnement de l'institution, mais qu'au contraire, elle en constitue un moment indispensable. Il faut donc pour Schelsky institutionnaliser la tension entre institutionnalisation et subjectivité. L'étape suivante de cette intégration fonctionnaliste de la subjectivité est la théorie des systèmes de Niklas Luhmann: l'idée luhmanienne de la "réduction de la complexité » est une reformulation de l'idée de « délestage ». Un autre type de réappropriation critique de la théorie des institutions se trouve dans le conservatisme de type libéral, qui considère que les valeurs humanistes sont un héritage culturel à préserver: ce courant de pensée reste hostile au normativisme, à l'universalisme, à la philosophie de l'histoire, à l'idée d'une fondation rationaliste du politique, mais considère que l'équilibre atteint en RFA doit être préservé, et qu'il est possible de développer dans ce contexte une culture bourgeoise. Il emprunte des éléments à Carl Schmitt et à Gehlen, mais les réoriente dans le sens d'une défense du système existant. Du point de vue des stratégies discursives, ce n'est sans doute pas un hasard si le conservatisme libéral s'articule à une interprétation de Hegel qui cherche à sortir de l'antagonisme entre hégélianisme de droite (à la Gehlen) et hégélianisme de gauche (théorie critique): Hegel serait celui qui, contre un rationalisme désincarné, aurait revalorisé les équilibres concrets existants, en évitant de faire de l'humanité le sujet de l'histoire (au profit du Weltgeist, c'est-à-dire du stade culturel et politique atteint à un moment donné). La pensée de Hegel fonderait donc le respect pour ce que la société a collectivement atteint.

$\mathrm{Si}$ on considère le diagnostic de Gehlen sur la culture de masse et sur le règne de la technocratie, indépendamment de son choix en faveur d'une éthique du pouvoir décomplexée, il est permis de se demander si Gehlen n'est pas finalement - dans la configuration actuelle - moins conservateur que les conservateurs libéraux qui sont le vrai visage du conservatisme contemporain, si on définit le conservatisme en termes relationnels. Le conservatisme apparaît alors comme le discours qui légitime les structures de domination existantes et discrédite idéologiquement toute tentative de 
remettre en cause les inégalités. En effet, Gehlen appartient comme Adorno à une tradition nietzschéenne de bourgeois antibourgeois, qui maintiennent une distance critique avec l'ordre existant, tandis que les conservateurs libéraux n'ont plus grandchose à y redire. Par ailleurs, il faut reconnaître à la méthode même de Gehlen d'avoir l'ambition de penser la société: il réfute l'idée - présente dans le conservatisme libéral - selon laquelle les sciences de la culture auraient une simple fonction de compensation face à la modernisation technique et économique, et ne seraient là que pour nous relier à la tradition. Comme le note Gérard Raulet, il y a de ce point de vue des « choses à tirer » des analyses de Gehlen, notamment de sa description de la société globalisée comme superstructure ${ }^{(37)}$.

Les conservateurs libéraux n'ont par ailleurs pas résolu le problème majeur de Gehlen: celui des critères normatifs permettant d'évaluer ou - le cas échéant - de critiquer les institutions. Comme le note Jens Hacke, ils sont en peine de définir précisément des notions telles que liberté, égalité, solidarité ${ }^{(38)}$. Il n'est donc pas certain que ce conservatisme libéral offre des instruments pour penser et contester les menaces qui pèsent sur la démocratie, et qu'a mises en évidence Thomas Piketty: l'évolution du capitalisme vers une société de rentiers, inégalitaire et potentiellement oligarchique ${ }^{(39)}$. Sans donc forcément raisonner en termes d'aliénation, et souscrire à un anti-capitalisme radical fondé sur une anthropologie utopique (pour reprendre ici les critiques de Gehlen envers la théorie critique), on peut se demander si face à cette évolution, le plaidoyer pour la modération, l'affirmation - teintée de scepticisme - des valeurs humanistes léguées par la tradition et l'attachement au «common sense » offrent les instruments pour comprendre ce processus et lui résister.

\section{Résumé}

Il s'agit dans cette contribution d'apporter un éclairage sur les évolutions du conservatisme en RFA, à travers l'exemple d'Arnold Gehlen. L'accent est mis sur la construction philosophique de ce conservatisme, dont l'arrière-plan est une anthropologie philosophique qui insiste sur l'instabilité structurelle de l'homme, qui fonde à son tour le besoin d'institutions stabilisatrices. Bien que la société de masse moderne corresponde pour lui à un déclin des institutions, Gehlen voit dans l'organisation technocratique un rempart contre la montée de l'individualisme et du narcissisme. Mais bien qu'il finisse par accepter le système politique et économique de la RFA, Gehlen ne peut toutefois pas être considéré comme un conservateur libéral.

\section{Zusammenfassung}

Im vorliegenden Beitrag geht es darum, anhand von Arnold Gehlen die Entwicklung des Konservatismus in der Bundesrepublik zu beleuchten. Der Akzent liegt auf der philosophischen Konstruktion dieses Konservatismus, dessen Hintergrund eine philosophische Anthropologie ist, die die strukturelle Instabilität des Menschen betont. Diese

37 Gérard Raulet, La philosophie allemande depuis 1945, Paris, Colin, 2006, p. 95.

38 Cf. J. Hacke, Philosophie der Bürgerlichkeit (note 9), p. 295.

39 Thomas Piketty, Le capital au XXI ${ }^{e}$ siècle, Paris, Seuil, 2013. 
Instabilität begründet ihrerseits die Notwendigkeit von stabilisierenden Institutionen. Obwohl die moderne Massengesellschaft in seinen Augen einem Zerfall der Institutionen entspricht, sieht Gehlen in der technokratischen Organisation einen Schutzwall gegen die Entwicklung des Individualismus und des Narzissmus. Wenn auch Gehlen schließlich das politische und wirtschaftliche System der Bundesrepublik akzeptiert hat, kann er nicht als ein Liberalkonservativer betrachtet werden.

\begin{abstract}
The present paper sheds light on the evolution of conservatism in the German Federal Republic, through the example of Arnold Gehlen. The focus is on the philosophical construction of this conservatism, whose background is a philosophical anthropology that emphasizes the structural instability of Man, which founds the need for stabilizing institutions. Although modern mass society corresponds for him to a decline of institutions, Gehlen sees technocratic organization as a bulwark against the rise of individualism and narcissism. Even if Gehlen finally accepted the political and economic system of the $B R D$, he can not be considered a liberal conservative.
\end{abstract}

